\title{
LA FIJACION DE OBJETIVOS EN LA DOCENCIA UNIVERSITARIA DE DISCIPLINAS ADMINISTRATIVAS: UN COMENTARIO
}

\author{
MARÍA CONCEPCIÓN VERONA MARTEL** \\ E-mail: cverona@defc.ulpgc.es
}

\begin{abstract}
RESUMEN
El presente trabajo tiene por objetivo hacer un comentario acerca de uno de los elementos clave de la estrategia docente, como es la fijación de los objetivos, dentro del marco de la enseñanza universitaria y con una especial referencia a las disciplinas administrativas. Asimismo, se destaca la importancia de que exista, dada la disciplina considerada, una estrecha relación entre universidad, sociedad y empresa.
\end{abstract}

Palabras claves: universidad, proceso enseñanza-aprendizaje, objetivos, disciplinas administrativas.

\begin{abstract}
The purpose of this paper is to show the importance of the objectives on the educationlearning process, with a special reference to the university teaching of the administrative disciplines. Likewise, we emphasize the importance of the existence of a narrow relation among University, society and firm.
\end{abstract}

Keywords: university, teaching-learning process, objectives, administrative disciplines.

\section{INTRODUCCIÓN}

La enseñanza superior es tarea que corresponde a la universidad, enseñanza que no se puede impartir sin considerar el entorno que le rodea; al contrario, debe tenerse muy presente, ya que la influencia que el entorno ejerce en la educación superior es considerable. Los factores sociales, culturales y económicos, las nue-

* Licenciada en Ciencias Económicas y Empresariales, Doctora en Ciencias Económicas y Empresariales. Actualmente ocupa el cargo de Profesora Titular de Universidad en la Universidad de Las Palmas de Gran Canaria en el área de Economía Financiera y Contabilidad. 
vas tecnologías, las regulaciones, son sólo algunos de los múltiples factores que inciden en la educación superior impartida en las universidades, y más aún en las materias que conforman las disciplinas administrativas.

La primera demanda a la universidad es el cumplimiento de su función de crear, desarrollar, transmitir y criticar la ciencia, la técnica y la cultura. Para ello, esta institución se convierte en depositaria del conocimiento al tiempo que es fiel a su mandato de transmitirla a la sociedad. Sin embargo, la hegemonía de la universidad como acumuladora de conocimientos requiere de un permanente ejercicio de transformación y acoplamiento a las nuevas demandas sociales.

Ortega y Gasset (1930) señala que se entenderá por universidad a:

«la institución en que se enseña al estudiante medio a ser un hombre culto y un buen profesional ... (pero) la universidad no puede ser eso sólo ... (pues) si la cultura y las profesiones quedaran aisladas en la universidad, sin contacto con la incesante fermentación de la ciencia, de la investigación, se anquilosarían muy pronto ... (con lo cual) la universidad es, además, ciencia. ... Pero además, ... la universidad tiene que estar también abierta a la plena actualidad; más aún, tiene que estar en medio de ella, sumergida en ella» ${ }^{1}$.

En este mismo sentido se expresa la Declaración Mundial sobre Educación Superior para el Siglo $\mathrm{XXI}^{2}$, que señala la necesidad de que las universidades atiendan las demandas sociales, culturales y educativas teniendo presente que la relevancia de la educación superior debe evaluarse según la correspondencia entre lo que la sociedad espera de las instituciones y lo que ellas hacen. Ello requiere visión ética, imparcialidad política, capacidad crítica y, al mismo tiempo, una mejor articulación con los problemas de la sociedad y del mundo del trabajo, basando las orientaciones a largo plazo en las necesidades y finalidades de la sociedad, incluyendo el respeto a la cultura y la protección ambiental.

El presente estudio tiene por objeto realizar un comentario acerca de los objetivos de la docencia universitaria, con especial referencia a las materias de las disciplinas administrativas, las cuáles han sufrido importantes cambios en las últimas décadas. Las universidades de todos los países han hecho grandes esfuerzos para dar respuesta a estos cambios siendo en algunos casos, la propia universidad el agente innovador y propulsor de ellos. El estudio de los objetivos tiene especial importancia, pues tal como afirma Mager ${ }^{3}$, la enseñanza será satisfactoria o eficaz en la medida en que consigue alcanzar los objetivos propuestos.

El presente trabajo se ha estructurado en cuatro secciones. Tras la introducción, en la sección segunda se hace referencia a la estrategia docente. La sección tercera es la que recoge los comentarios acerca de los objetivos de la docencia, elemento clave en toda estrategia docente. Por último, la sección cuarta está dedicada a las conclusiones.

\section{ASPECTOS GENERALES DE LA ESTRATEGIA DOCENTE}

Puede definirse la estrategia docente «como el modo en que el maestro utiliza la información, selecciona las fuentes y define el papel de los alumnos» ${ }^{4}$, incluyendo las prácticas específicas necesarias para dar cumplimiento al objetivo de la enseñanza. De esta forma, la estrategia docente es un plan global que trata de suscitar en el alumno el aprendizaje más efectivo posible, cuyo estudio abarca toda la gama de problemas que implica el proceso de enseñanza-aprendizaje. Su campo es muy amplio, comprendiendo, según Sawin $^{5}$,

«no sólo la forma de presentación de que hace uso el profesor, sino todos los actos que realiza relativos al favorecimiento de ciertas condiciones, agrupamiento de los alumnos, orientación, asignación de tareas y facilitación de orientación con miras al aprendizaje».

Con lo cual la estrategia docente hace referencia a todo el proceso a desarrollar para la correcta transmisión de los contenidos de una disciplina, esto es, una guía que orientará la práctica pedagógica y que contendrá respuestas a cuestiones como ¿qué enseñar?, ¿cómo hacerlo?, ¿cuándo es más adecuado?, y ¿cómo realizar la evaluación?.

De acuerdo con Ferrández ${ }^{6}$ el proceso instructivo, susceptible de ser considerado en toda estrategia docente, comprende los siguientes elementos: la fijación de objetivos; la planificación del proceso instructivo, que comprende, a su vez, una diversidad de elementos (contenidos objeto de aprendizaje, actividades que pro- 
fesor y alumno deberán realizar, material necesario para aprender dichos contenidos, conjunto de incentivos para motivar al alumno por el aprendizaje, y evaluación de resultados); y, por último, la comparación de los resultados obtenidos con los objetivos propuestos, con la consiguiente modificación del proceso en caso negativo (feed-back).

Por tanto, la estrategia docente puede ser vista como un plan en el que se han de fijar unos objetivos, detallar la programación que permitirá conseguirlos, las actividades necesarias para su cumplimiento, la/s metodología/s de enseñanza más adecuada/s, los recursos de apoyo a la docencia a disposición del profesor, y la forma de medir la consecución de los objetivos planteados.

Un esquema comprensivo de los factores más relevantes que intervienen en el proceso instructivo, susceptible de ser considerado en toda estrategia docente, se recoge en la Figura 1.

Hernández $z^{7}$ señala que para que este proceso educativo sea eficaz, tendría que caracterizarse, en líneas generales, por lo siguiente:

- Por la concreción y precisión de sus objetivos y procedimientos.
- Por su realismo y adecuación contextual.

- Por su flexibilidad, ante posibles alteraciones.

- Por la interrelación de todos los elementos integrantes del mismo.

En relación a este último aspecto, el citado autor señala que los elementos integrantes de la estrategia docente "no son entes aislados, formulados independientemente unos de otros, sino que la relación que existe entre ellos es tal, que no pueden subsistir unos sin los otros».

A raíz de todo lo expuesto y siguiendo a Hernández, para que el profesor realice una labor fructífera

«debe esforzarse por trazar en su tarea docente los contenidos a impartir (Currículum), de acuerdo con sus propias concepciones educativas (Principios educativos), partiendo de la realidad concreta de los alumnos y de sus circunstancias de aprendizaje (Diagnóstico inicial) y pretendiendo alcanzar unas determinadas metas u objetivos (Objetivos generales, específicos u operativos), a través de unos métodos, estrategias o recursos materiales específicos (Medios didácticos) $)^{8}$.

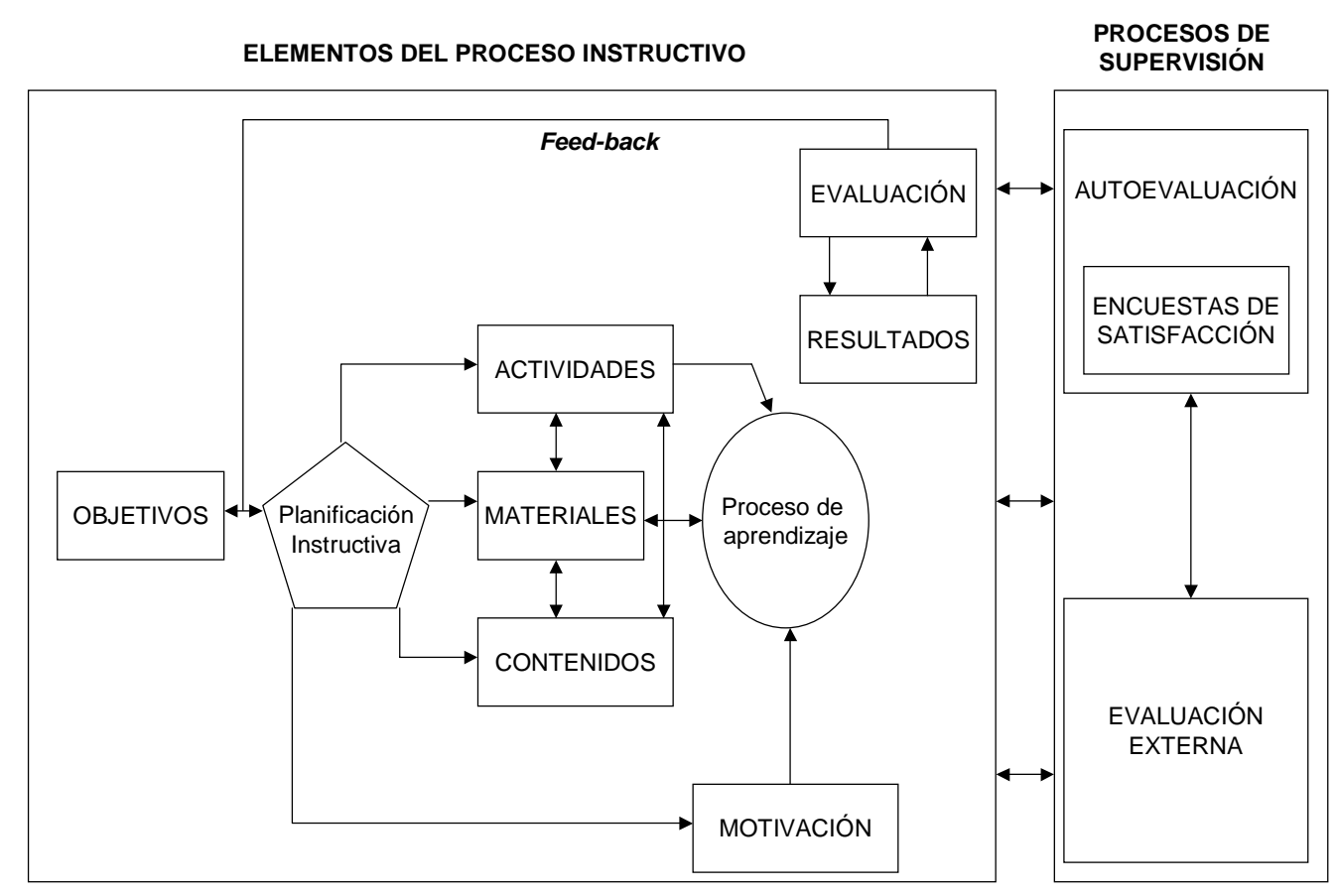

Fuente: adaptado por Dorta Velázquez (2003) de Ferrández et al. (1977).

Figura 1. Factores del proceso instructivo. 


\section{OBJETIVOS DE LA DOCENCIA DE LAS MATERIAS ADMINISTRATIVAS EN LA ENSEÑAZA UNIVER- SITARIA.}

El punto de partida de la estrategia docente lo constituye el establecimiento de los objetivos que se pretenden conseguir. Con ellos se pretende dar respuesta a cuestiones como ¿qué enseñar?, ¿qué se quiere que aprendan los alumnos?

Gronlund ${ }^{9}$ considera que los objetivos docentes son los resultados finales del aprendizaje, expresados en términos de transformación de la conducta del alumno. Para Peterssen ${ }^{10}$ el objetivo es

«la descripción de la conducta final del alumno, esperada o imaginada a priori por el profesor, conducta que se supone deberá ser capaz de demostrar el alumno después de recorrer el proceso de aprendizaje».

En términos similares se pronuncia Mager ${ }^{11}$ al considerar que el objetivo "es la descripción de la ejecución (realización de una actividad) que se pretende que los educandos estén en condiciones de realizar antes de que se les considere competentes. El objetivo describe un resultado previsto de la enseñanza, más bien que el proceso mismo de la enseñanza». Por tanto, para este autor el objetivo «denota qué se espera obtener de la asignatura (el resultado)».

Por su parte $A \mathrm{mat}^{12}$ señala que partiendo de la definición del término formar establecida por Beau ${ }^{13}$ se puede vislumbrar lo que son los objetivos de la formación. Beau indica que formar es «llevar al alumno de una situación inicial a una situación final».Y, como señala $\mathrm{Amat}^{14}$, la consecución de los objetivos permite al alumno alcanzar esa situación final deseada a la que se refiere Beau.

La concreción de los objetivos constituye uno de los mayores problemas que presenta el diseño de la estrategia docente, pero es al mismo tiempo uno de los aspectos que mejor pueden garantizar el éxito de la enseñanza. Tal como afirma Peterssen ${ }^{15}$, los objetivos son "factores decisivos para la elaboración de estrategias educativas con una finalidad racionaly, a lo que se puede añadir lo afirmado por Mager ${ }^{16}$ al señalar que "cuando faltan objetivos claramente definidos, se carece de una base sólida para seleccionar o preparar los materiales, el contenido o los métodos de la enseñanza». Asimismo, para Gimeno Sacristán ${ }^{17}$ explicitar los objetivos es una condición necesaria para que la técnica didáctica sea considerada científica.

Por ello, la fijación de los objetivos en el proceso de formación es fundamental, debiendo constituir la primera cuestión que ha de ser planteada al programar una asignatura. Éstos indicarán lo que el alumno debe conseguir al terminar su proceso de formación, con lo cual, los objetivos pueden ser vistos como metas en el aprendizaje. No es suficiente con que los objetivos estén en la «mente» de los profesores, sino que deben darse a conocer, ya que la exposición de los mismos no sólo es importante para el profesor sino, que es fundamental para los alumnos. A este respecto Mager ${ }^{18}$ comenta que si los alumnos tienen ante la vista objetivos claros no tendrán "necesidad de tantear y 'adivinar' las intenciones del profesor», con lo cual una formulación clara de objetivos revierte en bien del alumno, pues éste «tiene a su disposición los medios para organizar sus esfuerzos hacia la consecución de aquellos objetivos».

Peterssen ${ }^{19}$ señala que el establecimiento de los objetivos de la docencia de una disciplina es una tarea de enorme importancia, ya que:

- Permite una planificación de la enseñanza.

- Obliga al profesor a programar con precisión las actividades a desarrollar para conseguir que se logren los objetivos.

- Hace posible una evaluación formativa, basada en criterios fijados con anterioridad.

- Permite comprobar y corregir la instrucción en su conjunto.

- Garantizar una revisión constante de los planes de enseñanza, esto es, de la planificación y ejecución de la enseñanza.

- Facilita la comunicación entre profesores y estudiantes, favoreciendo la relación educativa.

Para el citado autor, la importancia del establecimiento de objetivos radica en que

"permite planificar, realizar y evaluar la enseñanza según criterios racionales sin que se pierdan de vista la espontaneidad y la decisión simultáneas, cuando tienen sentido, para que la enseñanza pueda, de hecho, lograr su finalidad: que los alumnos tengan éxito en su

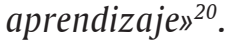


En líneas similares se pronuncia Mager ${ }^{21}$ al señalar que el establecimiento de los objetivos nos ofrecen una base sólida para seleccionar o plantear el contenido y los procedimientos de la enseñanza; evaluar o determinar el éxito de la enseñanza ${ }^{22}$; y, organizar los esfuerzos y actividades de los alumnos con vistas a realizar los fines fundamentales de la enseñanza.

Por otro lado Millar ${ }^{23}$, al hacer referencia a los objetivos, señala que las cualidades básicas que han de reunir los objetivos son: ser reales y comprensibles; fundamentarse en las necesidades educativas de los estudiantes; ser auténticamente factibles, es decir que se puedan llevar a cabo; y ser mensurables, para que su evaluación pueda ser objetiva.

Por su parte, Hernández ${ }^{24}$ señala que para que los objetivos fijados sean adecuados y realistas han de ser el resultado de lo que queremos lograr (principios educativos); la realidad predominante (diagnóstico inicial ${ }^{25}$ ); y el propio currículum (contenido).

Por otro lado, Amat ${ }^{26}$ señala que para que los objetivos sean útiles al diseñar, impartir y evaluar un programa, han de ser definidos tanto para el conjunto de la asignatura como para cada sesión o parte de ella; alcanzables de acuerdo con el nivel de los alumnos, el tiempo disponible y los medios de los que se dispone; contrastados al fijarlos para comprobar que son útiles y coherentes con lo que se necesita; y evaluados durante y al finalizar la acción de formación.

En todas las disciplinas los objetivos, deberían estar fijados de forma que ayudasen a los estudiantes "a aprender a aprender», a pensar y a ser creativos. Se requiere de programas de enseñanza flexibles que permitan introducir los cambios que se produzcan en las necesidades y demandas de la sociedad sería lo más recomendable para las universidades ${ }^{27}$. Por eso, toda reforma de los planes de estudio debería estar guiada por una serie de principios generales entre los que se han de encontrar:

- La actualización de los contenidos y de las enseñanzas a impartir.

- La flexibilización de las enseñanzas.

- El cambio en la metodología de la enseñanza (reducir el predominio de clases magistrales y fomentar aquellos métodos que requieran la participación activa del alumno).
- La necesidad de vincular la universidad y la sociedad.

Por tanto, acerca de los objetivos podemos concluir, tomando las palabras de Mager ${ }^{28}$ que los objetivos son instrumentos útiles para la programación, la realización y la evaluación de la enseñanza; son útiles para indicar el contenido y los procedimientos que permitirán al profesor desarrollar una enseñanza eficaz, y le ayudará tanto a organizar el proceso mismo de la enseñanza como en la tarea de preparar los medios para descubrir si se ha obtenido el éxito con la enseñanza impartida. Además, si el profesor hace los objetivos asequibles a los alumnos, se podrá eliminar "la pérdida de energías que supone forzarles a que adivinen cuáles deberán ser los resultados principales de la enseñanza».

Dado lo expuesto, se puede afirmar que disponer de unos objetivos fijados de forma explícita, con claridad y precisión, proporciona tanto al profesor como al alumno una guía de enorme valor en el proceso enseñanza-aprendizaje, con lo cual la formulación de objetivos debe ser la primera tarea a desarrollar para planificar racional y adecuadamente un proceso educativo. Sin embargo, no es una tarea fácil, debido a que no existe un único objetivo, sino que se pueden señalar distintos tipos de objetivos cuya definición comporta distintos niveles de complejidad y amplitud. Así, al fijar los objetivos se debe ir de los más generales y de más largo plazo a los más específicos y de corto plazo, organizándolos de tal manera que la consecución de los objetivos de los escalones inferiores facilite alcanzar los de los escalones superiores.

Una forma de clasificar los objetivos a perseguir en el proceso de formación de un alumno universitario es la que los agrupa en ${ }^{29}$ :

- Objetivos generales. Son amplios, complejos y alcanzables en un período de tiempo relativamente largo. De acuerdo con Rotger ${ }^{30}$, se trata de las formas de conocimiento, actitudes, hábitos y habilidades que, de una manera general, hay que inculcar en el alumno a través del proceso de aprendizaje, y que son comunes a todos los niveles, áreas de conocimiento y campos de un plan de estudios.

- Objetivos concretos, específicos y operativos. Son alcanzables a medio o corto plazo. Concretamente, los objetivos específicos consti- 
tuyen el resultado de una experiencia de aprendizaje completa y alcanzable a medio plazo; hacen referencia a los productos peculiares de cada asignatura. Los objetivos operativos son el resultado de un aprendizaje concreto cuya consecución puede realizarse a corto plazo; representan la aplicación de los objetivos específicos a las unidades temáticas concretas del programa de la asignatura, en función de las características de los alumnos y de los recursos disponibles.

El paso desde los objetivos generales a los objetivos específicos, y de éstos a los objetivos operativos, permite la concreción en el proceso de establecimiento de objetivos ${ }^{31}$.

Los objetivos generales pueden admitir varias interpretaciones, por lo que en su formulación no constan manifestaciones observables que permitan evaluarlos sin ambiguiedad. Por el contrario, los objetivos específicos, dada su manifestación concreta, son observables, medibles y evaluables ${ }^{32}$. Así, el proceso de fijación de objetivos no debe quedar limitado a la especificación de las metas a alcanzar a corto plazo, en cuanto a conocimientos y actitudes respecto a un tema, unidad de trabajo o disciplina que puedan ser enseñados, sino que es necesario, además, lograr la congruencia con objetivos a más largo plazo e incluso tener en cuenta que los objetivos y los contenidos deben estar en consonancia con la filosofía y objetivos globales del sistema educativo. Pero también es preciso considerar que aunque los objetivos generales proporcionan un marco de referencia útil para la planificación de la materia a impartir, su generalidad puede ocultar el diseño de acciones precisas y claras que permitan asegurar una progresión en el aprendizaje o establecer el óptimo aceptable en el logro de los objetivos; por ello, junto a estos objetivos generales, se han de formular los objetivos propios de las asignaturas.

En general, para cualquier disciplina universitaria Romero Quintero ${ }^{33}$ señala que se podrían considerar apropiados los objetivos generales siguientes:

- Interesar al alumno por la materia impartida, en la cual puede profundizar haciendo uso de la documentación que contiene el programa.

- Permitir que el alumno adquiera aquellos conocimientos más relevantes y adecuados, teniendo en cuenta las limitaciones temporales, de métodos y medios que puedan existir.
- Favorecer el establecimiento de una relación provechosa, en la que el alumno se convierta en un elemento activo dispuesto a buscar soluciones a los problemas.

- Fomentar la inquietud del alumno en relación a una posterior labor de investigación.

En lo que a nuestra disciplina se refiere, antes de señalar de forma más concisa los objetivos que se persiguen, es preciso hacer referencia a ciertos aspectos. Así, por ejemplo, una de las características que poseen las materias de administración es su relación directa con la práctica profesional, frente a otras materias cuyos objetivos son únicamente formativos o instrumentales. En este sentido, el profesor universitario que imparta materias de nuestra disciplina no puede quedarse en el campo del deber ser, sino que debe conocer también el ser de la realidad, e inculcar además el comportamiento moral exigible al profesional de esta disciplina.

Obviamente, la enseñanza universitaria de la disciplina a la que se refiere este trabajo no puede pretender formar a los futuros profesionales mediante un conjunto de recetas prácticas que respondan a la realidad económica del momento; al contrario, debe dotar al alumno de la capacidad de emitir criterios propios debidamente fundamentados tanto frente a la realidad actual, como ante los cambios económicos y sociales que se experimentarán en el futuro. En ningún libro se podrían recoger los múltiples problemas que se le presentan a cada empresa particular a lo largo de su vida, por ello es necesario "conseguir del alumno una formación teórica que le forme para resolver los innumerables casos prácticos de la economía de la empresa, incluso aquellos problemas nuevos que nunca se le habían presentado», como señalaba Schmalenbach $^{34}$ en su trabajo de 1934.

En la docencia de las materias propias de nuestra disciplina el objetivo global que se persigue es proporcionar al alumno una efectiva formación profesional en los temas fundamentales de economía de la empresa, así como de la organización, gestión y dirección empresarial, creando las condiciones que le permitan formar su especialización, ser consciente del carácter dinámico de la misma así como del papel que desempeña en la sociedad. Por tanto, cuando un alumno termina sus estudios en esta disciplina, debe estar capacitado para cubrir la demanda social en su área de especialización; adaptarse a los cambios (tecnológicos, etc.) que pue- 
dan surgir en el desempeño de su profesión; y desarrollar una actitud crítica y creativa que le posibilite solucionar los problemas que surjan en su vida laboral.

Para ello, el alumno debe adquirir un conjunto de conocimientos y desarrollar una serie de habilidades y actitudes. Entre los primeros, debe tener conocimiento de la terminología, conceptos, principios y aplicaciones básicas de su disciplina, y ser capaz de interrelacionar las distintas materias adquiridas en su formación. En relación a las segundas, el alumno debe saber manejar los métodos e instrumentos propios de su disciplina, aplicar los conocimientos teóricos para solucionar problemas reales, valorar el trabajo interdisciplinario, tener interés por una formación permanente, ser consciente de la responsabilidad que asume en el desempeño de su labor, entre otros.

A este respecto, se pueden mencionar los principios de la didáctica que Tonne ${ }^{35}$ en 1961 señalaba para las materias económico-empresariales, y que aún hoy siguen siendo válidas:

- Alcanzar una comprensión profunda de la naturaleza general del mundo de los negocios.

- Proporcionar formación en fases especializadas de la actividad empresarial.

- Proporcionar la formación necesaria para la dirección de empresas.

- Asentar los valores culturales y éticos para el desarrollo de las tres metas anteriores.

Por su parte, Díez de Castro ${ }^{36}$ señalan que se pueden distinguir cinco objetivos principales para las materias de Administración de Empresas, a saber:

- Estudio de los conocimientos acumulados y de la información necesaria para el desarrollo de la preparación y capacidades de los alumnos.

- Estimular en los alumnos una posición crítica sobre los conocimientos disponibles.

- Desarrollar las habilidades analíticas y de toma de decisiones del alumno.

- Desarrollo de aptitudes de comportamiento para la resolución de problemas.

- Inculcar en los alumnos actitudes y valores que permitan el desarrollo de un comportamiento ético en el mundo de los negocios.
En este sentido también se pueden recoger las palabras de Déniz Mayor ${ }^{37}$, que aunque enunciadas expresamente para la enseñanza universitaria de la Contabilidad, son extrapolables y adaptables para todas las materias de las disciplinas administrativas. Así, según este autor, el objetivo fundamental de la educación universitaria de una disciplina concreta consiste en que el estudiante adquiera una sólida formación básica acerca de la naturaleza, el papel y los fundamentos teóricos de la misma, desarrolle su capacidad de juicio para enfrentarse a los problemas, entienda y sepa aplicar los métodos y procedimientos técnicos, y quede capacitado para desarrollar nuevos conocimientos o adaptar adecuadamente los ya existentes para satisfacer las necesidades de información de una sociedad en permanente cambio; sin olvidar mantener siempre un comportamiento ético en todas las actividades y operaciones que realice a lo largo de su vida profesional.

En este punto, y dado que junto con los alumnos es el profesor el otro elemento humano fundamental en la universidad, se pueden recoger las palabras de Tua Pereda ${ }^{38}$ relativas a algunos de los aspectos que el profesor ha de tener presente al desempeñar la actividad docente. Este autor concretamente se refiere a la docencia de la disciplina contable, pero consideramos que también se pueden aplicar a la docencia de la disciplina que nos ocupa:

«En primer lugar, razonando más desde la relatividad del conocimiento que desde el dogma, sembrando inquietudes y atendiendo más al fondo que a la forma, es decir, dando prioridad a los fundamentos sobre la técnica; pero, además, explicando no sólo los hechos sino también su origen, su evolución y sus consecuencias; siendo conscientes de la situación actual de nuestra disciplina, como preludio del inmediato futuro, potenciando los planteamientos interdisciplinares; sensibilizando a nuestros alumnos hacia la investigación, desarrollando en definitiva, el criterio y la capacidad de juicio de quienes hoy están en las aulas y en un futuro no muy lejano estarán inmersos en un mundo profesional de cambio constante».

La disciplina administrativa al igual que las restantes disciplinas debe -por encima de todos los objetivos asignados a la universidad- perseguir y adaptarse a las exigencias de la sociedad y, especialmente, a las del mundo empresarial. Este objetivo es vital por cuanto del mismo parte la 
necesidad de revisar periódicamente el cuerpo de conocimientos que constituyen el programa de cada asignatura (Aguiar Díaz) ${ }^{39}$.

De ahí que sea necesario reforzar la relación entre universidad y el sector empresarial para que la formación de los universitarios responda a las necesidades de una sociedad avanzada y moderna. Los empresarios demandan que la tarea de la universidad sea formar a los alumnos para el trabajo, siendo la opinión más generalizada entre los empresarios que los universitarios padecen de una sobre educación con falta de conocimientos prácticos. Sin embargo, aunque, evidentemente, la preparación profesional de los estudiantes universitarios es una de las tareas primordiales de la universidad no es la única, porque su cometido es más amplio. La solución a esta divergencia de opiniones según Michavila $^{40}$ es que

"para el desarrollo de la sociedad del conocimiento se requiere un acercamiento entre universidad y sociedad productiva, sin que ninguna de ellas renuncie a su esencia y a sus fines. Este acercamiento tiene una vertiente formativa (capacitación de los titulados para el mundo del trabajo, formación permanente) y otra de colaboración (investigación, desarrollo, creación de riqueza, desarrollo regional)».

Así como, que

«las empresas deben superar la tradicional desconfianza hacia la educación superior y comprender que la función de la universidad no es preparar titulados a medida de sus exigencias, sino dar una formación superior sólida en actitudes y aptitudes a los futuros trabajadores y directivos. ... Las empresas ... deben vencer los perjuicios que las alejan de las instituciones universitarias. Es deseable que formulen demandas claras a las instituciones de educación superior, que favorezcan la movilidad de profesores hacia la industria, que compartan responsabilidades en el diseño y seguimiento de currículos académicos y que transmitan a la universidad sus propios valores. Los acuerdos entre universidad y empresa deben incrementarse y fundarse en bases de confianza $y$ beneficios mutuos».

Todo ello supone que la labor docente persiga una formación integral de los alumnos, siendo responsabilidad del profesor universitario (y de los estudiantes), formar (formarse como) titu- lados que, conociendo cuál es la realidad empresarial de su país, sepan adaptarse a ella para transformarla, con el fin último de elevar los niveles de desarrollo social y económico del país. Sin embargo, la universidad no ha de limitarse a fabricar profesionales, pues su objetivo, como ya se ha mencionado, es más amplio: formar personas que, como parte de su vida, desarrollen una actividad profesional, adquiriendo además otras capacidades que le permitan aprender por sí mismos, evolucionar y desarrollarse tanto como profesionales, así como seres humanos.

Una vez que se han definido los objetivos, el paso siguiente es determinar los métodos y recursos para poder alcanzarlos. Sólo eligiendo los más adecuados, no cualquiera de ellos, se podrán alcanzar los objetivos fijados. A su vez, la evaluación aportará información de lo que ha acontecido en el proceso enseñanza-aprendizaje, permitiendo comprobar si tanto los objetivos previstos como la metodología que se ha empleado, han sido los adecuados.

\section{CONCLUSIONES}

El presente trabajo pretende hacer un comentario de los aspectos más relevantes de un elemento clave de la estrategia docente como es la fijación de los objetivos a conseguir en el proceso enseñanza-aprendizaje, con una especial referencia a la docencia universitaria de las materias de las disciplinas administrativas. Acerca de los objetivos podemos concluir que su fijación de forma clara y concisa es imprescindible para que la enseñanza se realice de forma eficaz; el profesor ha de realizar ese esfuerzo en aras de que su labor docente se realice de forma correcta y con éxito; su interés, su formación y su experiencia le serán de gran ayuda en el establecimiento de los mismos.

Al mismo tiempo, cuando se está analizando esta disciplina, es preciso hacer referencia a la estrecha vinculación que debe existir entre universidad y empresa, pues es responsabilidad de la universidad "formar» profesionales capaces de desenvolverse eficazmente en el complejo mundo de la empresa.

\section{NOTA}

1 Ortega y Gasset, J. Misión de la Universidad. Madrid, Editorial Revista de Occidente, 1930, p.

2 UNESCO, 1998. 
3 Mager, R. F. Formulación operativa de los objetivos didácticos. Madrid, Editorial Marova, 1980, p.

4 Guerlach, V. y Ely, D. Tecnología didáctica. Buenos Aires, Ed. Paidós, 1979, Pág.

5 Sawin, E.I. Técnicas básicas y educación. Madrid, Editorial Magisterio Español, 1971, p.

6 Ferrández, A. y Sarramona, J. Quinta edición. La educación. Constantes y problemática actual. Barcelona, Editorial CEAC, 1977, p.

7 Hernández, P. Diseñar y enseñar. Teoría y técnicas de la programación y del proyecto docente. Madrid, Editorial Narcea e ICE Universidad de la Laguna, 1989, p.

8 Op. Cit. Pág.

9 Gronlund, N.E. Measurement and evaluation of teaching. New York, Ed. The McMillan Co., 1971, p.

10 Peterssen, W. H. La enseñanza por objetivos de aprendizaje: fundamentos y práctica. Madrid, Editorial Santillana, 1976.

11 Mager. Op. cit.

12 Amat, O. Aprender a enseñar. Una visión práctica de la formación de formadores. Barcelona, Editorial Gestión 2000, 2002.

13 Beau, D. 100 fiches de pédagogie des adultes. París, Ed. Les Editions d'Organisation, 1976.

14 Amat. Op. cit.

15 Peterssen. Op. cit.

16 Mager. Op. cit.

17 Gimeno Sacristán, J. Teoría de la enseñanza y desarrollo del curriculo. Salamanca, Ed. Anaya, 1981.

18 Mager. Op. Cit.

19 Peterssen. Op. Cit.

20 Peterssen. Op. Cit.

21 Mager. Op. Cit.

22 Los exámenes o pruebas formulados para determinar en qué medida se han alcanzado resultados didácticos de importancia sólo podrán seleccionarse o crearse inteligentemente cuando dichos objetivos se han expresado de manera explícita (Mager, 1980).

23 Millar, H. Medical Education and Medical Research.Ciudad, The Lancet, enero de 1971, pp. 1-6.

24 Hernández. Op. Cit.

25 Para Hernández (1989), el diagnóstico inicial es un requisito previo ineludible, no pudiéndose trazar unos objetivos, ni los medios a emplear sin realizar el mismo con antelación. Este diagnóstico inicial, según el autor, es el que nos puede ayudar a que la labor educativa se ajuste a las necesidades y condiciones de la realidad concreta, donde se realiza el proceso de enseñanza-aprendizaje; el análisis de la situación del centro, del alumno y del contexto permitirá delimitar la acción educativa.

26 Amat. Op. Cit.

27 Comisión Bedford de la American Accounting Association (AAA, 1986)

28 Mager. Op. Cit.

29 En el trabajo de Bierza (1980) y De Juan Herrero (1995) se puede encontrar un estudio más amplio acerca de la clasificación de objetivos atendiendo a distintos criterios. Asimismo, el primero recoge también las distintas denominaciones que se han utilizado en diferentes estudios para hacer referencia a los objetivos de la docencia.

30 Rotger, B. El proceso programador en la escuela. Teoría y práctica de la programación didáctica. Madrid, Editorial Escuela Española, 1985.

31 Hernández. Op. Cit.
32 Morales Vallejo, P. Los objetivos didácticos, Cuadernos monográficos del ICE. Bilbao, Universidad de Deusto, 1995.

33 Romero Quintero, L. Proyecto docente. Gran Canaria - España, Universidad de Las Palmas, 1997.

34 Citado por Schneider, E. Contabilidad industrial. Fundamentos y principales problemas. Madrid, Editorial Aguilar, 1959.

35 Tonne, H. A. Principles of Business Education. New York, Ed. McGraw Hill, 1961.

36 Díez de Castro, E. P.; Leal Millán, A. y Martín Jiménez, F. La enseñanza de Administración de Empresas. En Revista de Economía y Empresa, vol. 10, № 24-25, 1989, pp. 159-175.

37 Déniz Mayor, J. J. Proyecto docente. Gran Canaria - España, Universidad de Las Palmas, 2003.

38 Tua Pereda, J. En torno a la docencia de la Contabilidad: Una reflexión personal. En Revista Técnica Contable, vol. 47, $\mathrm{N}^{\circ}$ 557, 1995, pp. 313-334, 350.

39 Aguiar Díaz, I. Proyecto docente. Gran Canaria - España, Universidad de Las Palmas.

40 Michavila, F. y Calvo, B. La universidad española hacia Europa. Madrid, Ed. Fundación Alfonso Martín Escudero, 2000.

\section{REFERENCIAS BIBLIOGRÁFICAS}

Aguiar Díaz, I. Proyecto docente. Gran Canaria España, Universidad de Las Palmas de Gran Canaria, 1990.

Amat, O. Aprender a enseñar. Una visión práctica de la formación de formadores. Barcelona, Ed. Gestión 2000, 2002.

American Accounting Association. Committee on the Future Structure, Content and Scope of Accounting Education (The Bedford Committee). Future Accounting education: Preparing for the expanding profession. En Issues in Accounting Education, Spring 1986, vol. 1, pp. 168-195.

Beau, D. 100 fiches de pédagogie des adultes. París, Ed. Les Editions d'Organisation, 1976.

Bircea, C. Hacia una didáctica por objetivos. Madrid, Ed. Morata, 1980.

Déniz Mayor, J. J. Proyecto docente. Gran Canaria - España, Universidad de Las Palmas, 2003.

Dorta Velázquez, J. A. Proyecto docente. Gran Canaria - España, Universidad de Las Palmas, 2003.

Díez de Castro, E. P.; Leal Millán, A. y Martín Jiménez, F. La enseñanza de Administración de Empresas. En Revista de Economía y Empresa, vol. 10, № 24-25, 1989, pp. 159-175.

Ferrández, A. y Sarramona, J. Quinta edición. La educación. Constantes y problemática actual. Barcelona, Ed. CEAC, 1997.

Guerlach, V. y Ely, D. Tecnología didáctica. Bue- 
nos Aires, Ed. Paidós, 1979.

Hernández, P. Diseñar y enseñar. Teoría y técnicas de la programación y del proyecto docente. Madrid, Ed. Narcea e ICE Universidad de la Laguna, 1989.

Gimeno Sacristán, J. Teoría de la enseñanza y desarrollo del curriculo. Salamanca, Ed. Anaya, 1981.

Gronlund, N.E. Measurement and evaluation of teaching. New York, Ed. The McMillan Co., 1971.

De Juan Herrero, J. Introducción a la enseñanza universitaria. Didáctica para la formación del profesorado. Madrid, Ed. Dykinson, 1995.

Mager, R. F. Formulación operativa de los objetivos didácticos. Madrid, Ed. Marova, 1980.

Michavila, F. y Calvo, B. La universidad española hacia Europa. Madrid, Ed. Fundación Alfonso Martín Escudero, 2000.

Millar, H. Medical Education and Medical Research. En The Lancet, enero de 1971, pp. 1-6.

Morales Vallejo, P. Los objetivos didácticos. En Cuadernos monográficos del ICE. Bilbao, Universidad de Deusto, 1995.
Ortega y Gasset, J. Misión de la Universidad. Madrid, Ed. Revista de Occidente, 1930.

Peterssen, W. H. La enseñanza por objetivos de aprendizaje: fundamentos y práctica. Madrid, Ed. Santillana, 1976.

Romero Quintero, L. Proyecto docente. Gran Canaria - España, Universidad de Las Palmas, 1997.

Rotger, B. El proceso programador en la escuela. Teoría y práctica de la programación didáctica. Madrid, Ed. Escuela Española, 1985.

Sawin, E.I. Técnicas básicas y educación. Madrid, Ed. Magisterio Español,1971.

Schneider, E. Contabilidad industrial. Fundamentos y principales problemas. Madrid, Ed. Aguilar, 1959.

Tonne, H. A. Principles of Business Education. New York, Ed. McGraw Hill, 1961.

Tua Pereda, J. En torno a la docencia de la Contabilidad: Una reflexión personal. En Revista Técnica Contable, vol. 47, $\mathrm{N}^{\circ}$ 557, 1995, pp. 313-334, 350.

UNESCO. Declaración Mundial sobre Educación Superior para el Siglo XXI. París, Conferencia Mundial sobre Educación Superior, 1998. 